This is a preprint of an article published in Synthese. The final authenticated version is available online at https://doi.org/10.1007/s11229-020-02660-w

\title{
Intellectual Generosity \& The Reward Structure Of Mathematics
}

\author{
Rebecca Lea Morris
}

August 7, 2020

\begin{abstract}
Prominent mathematician William Thurston was praised by other mathematicians for his intellectual generosity. But what does it mean to say Thurston was intellectually generous? And is being intellectually generous beneficial? To answer these questions I turn to virtue epistemology and, in particular, Roberts and Wood's (2007) analysis of intellectual generosity. By appealing to Thurston's own writings and interviewing mathematicians who knew and worked with him, I argue that Roberts and Wood's analysis nicely captures the sense in which he was intellectually generous. I then argue that intellectual generosity is beneficial because it counteracts negative effects of the reward structure of mathematics that can stymie mathematical progress.
\end{abstract}

\section{Contents}

1 Introduction 1

2 Virtue Epistemology 2

3 William Thurston $\quad 4$

4 Reflections on Thurston \& Intellectual Generosity 10

5 The Benefits of Intellectual Generosity: Overcoming Problems with the $\begin{array}{ll}\text { Reward Structure of Mathematics } & 13\end{array}$

6 Concluding Remarks

\section{Introduction}

William Thurston (1946 - 2012) was described by his fellow mathematicians as an intellectually generous person. This raises two questions: (i) What does it mean to say that Thurston was intellectually generous?; (ii) Is being intellectually generous beneficial? To answer these questions I turn to virtue epistemology. I answer question (i) by showing that Roberts and Wood's (2007) analysis nicely captures Thurston's intellectual generosity. To answer question (ii) I first show that the reward structure of mathematics can hinder 
This is a preprint of an article published in Synthese. The final authenticated version is available online at https://doi.org/10.1007/s11229-020-02660-w

mathematical progress. I then argue that intellectual generosity is beneficial because it can ameliorate problems caused by the reward structure and thereby promote mathematical discoveries.

I start in section (2) by presenting some background on virtue epistemology and briefly sketching the analysis of intellectual generosity developed by Roberts and Wood. In section (3), I describe two contrasting episodes from Thurston's career: his work on foliation theory and the geometrization conjecture. I then argue that Thurston was intellectually generous, in Roberts and Wood's sense, when working on the geometrization conjecture. To do this, I draw heavily from Thurston's essay "On proof and progress in mathematics," as well as on email interviews I conducted with a number of research mathematicians who knew and worked with him. In section (4) I address some critical questions about intellectual generosity, both in the abstract and about Thurston's exemplification of it. In section (5) I argue that the reward structure of mathematics can stymie mathematical progress in two ways: (i) in certain circumstances it can lead to the abandonment of entire fields of mathematics that could still be fruitfully explored; (ii) it strongly disincentivizes mathematicians from making their work accessible. I then argue that intellectual generosity is beneficial because, by cultivating it, mathematicians can counteract the negative influences of the reward structure and thus continue to make important mathematical discoveries. Finally, in section (6), I point out potentially fruitful avenues of future work on intellectual generosity in mathematics.

\section{Virtue Epistemology}

\subsection{Background Context}

Traditionally, epistemology has been concerned with evaluating beliefs. Virtue epistemology, however, is concerned with evaluating agents via their intellectual virtues and vices (Battaly, 2008, sec.1). At a high level, "intellectual virtues are characteristics that promote intellectual flourishing, or which make for an excellent cognizer" (Turri et al., 2018). For virtue reliabilists such as Ernest Sosa (1991a,b) and John Greco (2002), these characteristics are reliable faculties, such as memory and perception. For virtue responsibilists like Linda Zagzebski (1996) and Robert Roberts and W. Jay Wood (2007), these characteristics are not faculties, but rather character traits such as intellectual humility and generosity. A complete account of intellectual virtue, however, will likely need to incorporate both kinds of intellectual virtues (see e.g. Battaly $(2008,651)$ ).

Although virtue responsibilists agree that intellectual virtues are character traits, they disagree about the nature of the relationship between intellectual virtues and more traditional epistemological questions about knowledge. Conservative virtue responsibilists, like Zagzebski, use intellectual virtues to tackle issues of traditional epistemological concern, such as defining knowledge and solving the Gettier problem (Baehr, 2008, 472). Autonomous virtue responsibilists, like Roberts and Wood, are interested in the intellectual virtues in their own right, independent from any connection they may have to more traditional epistemological concerns (Baehr, 2008, 473).

Virtue responsibilists have begun to investigate a variety of individual intellectual virtues, with intellectual humility, intellectual courage and epistemic justice being among the most 
This is a preprint of an article published in Synthese. The final authenticated version is available online at https://doi.org/10.1007/s11229-020-02660-w

popular (Turri et al., 2018, §10.2). ${ }^{1}$ Intellectual generosity, however, is an example of a virtue that "[has] received less attention to date, though not for lack of philosophical merit" (Turri et al., 2018, §10.2). Fortunately Roberts and Wood (2007, chp.11) devoted a rich chapter in their book to analyzing this virtue.

\subsection{Roberts and Wood's Account of Intellectual Generosity}

Roberts and Wood aimed to clarify the intellectual virtues in their 2007 book Intellectual Virtues: An Essay in Regulative Epistemology. However, they did not attempt to do this by offering definitions in the form of necessary and sufficient conditions. Instead, they provided a conceptual "map" of the virtues (Roberts and Wood, 2007, 23-30). In their chapter on intellectual generosity they used case studies from the history of science to help achieve this.

As generosity intuitively involves giving valuable things to others, Roberts and Wood first identified the kinds of "things" that can be given in intellectual life. They distinguished two kinds of intellectual goods: intrinsic and extrinsic (Roberts and Wood, 2007, 292). Intrinsic intellectual goods are things like knowledge and understanding which are internal to an intellectual practice. Extrinsic intellectual goods are things like fame, position or rank and prizes which are external to an intellectual practice. ${ }^{2}$

Roberts and Wood then presented a number of case studies to exemplify intellectual generosity. I'll review one of their case studies here: that of Barbara McClintock. (Roberts and Wood, 2007, 298-301). McClintock was a cytogeneticist at Cornell, studying the role chromosomes play in inheritance. Roberts and Wood pointed to two indications of her intellectual generosity: (i) a concern for the intellectual well-being of her students over and above a concern for her own extrinsic intellectual goods; (ii) a concern for intrinsic intellectual goods over and above a concern for her own extrinsic goods.

To illustrate McClintock's concern for the intellectual well-being of her students over concern for her own extrinsic intellectual goods, Roberts and Wood referred to her groundbreaking work showing that genes are located on chromosomes (Roberts and Wood, 2007, 298-299). Instead of working on this project alone so as to receive sole credit, McClintock recruited Harriet Creighton, a graduate student at Cornell, to work with her. The pair then wrote up the results in their paper "A correlation of cytological and genetical crossing-over in Zea mays" (Creighton and McClintock, 1931) for which they both received world wide recognition. McClintock's gift of this research project to Creighton followed the policy, due to Cornell geneticist Rollins Emerson, of giving important research to students. Roberts and Wood noted that this policy came from "a concern for the well-being of one's students and a willingness to give them some of the best things the profession has to offer, both internal and external to its practices" (Roberts and Wood, 2007, 299).

To illustrate McClintock's concern for intrinsic intellectual goods over concern for her own extrinsic intellectual goods, Roberts and Wood pointed to two episodes later in her

\footnotetext{
${ }^{1}$ For work on intellectual humility see e.g. Whitcomb et al. (2017), Church (2016), Roberts and Wood (2007, chp.9). For work on intellectual courage, see e.g. Baehr (2011), Roberts and Wood (2007, chp.8). For work on epistemic justice, see e.g. Fricker (2007), Medina (2011).

${ }^{2}$ We will see that intellectual generosity involves a concern with intrinsic over extrinsic intellectual goods. However I am agnostic on the question whether intrinsic or extrinsic goods are ultimately superior.
} 
This is a preprint of an article published in Synthese. The final authenticated version is available online at https://doi.org/10.1007/s11229-020-02660-w

career. First, in the 1950s, when other scientists became disinterested in her work, McClintock did not view it as a set back like she would if she were concerned with her own extrinsic intellectual goods. Instead she used the time to listen to developments and breakthroughs in the field made by other researchers thus indicating her concern with the intrinsic intellectual goods (Roberts and Wood, 2007, 300-301). As another example, Roberts and Wood reported that when she received extrinsic intellectual goods, such as the Nobel Prize in 1983, she viewed it "not as an unmixed blessing" (Roberts and Wood, 2007, 301) due to the distraction it caused from her pursuit of intrinsic intellectual goods.

Roberts and Wood concluded the chapter by offering the following characterization of intellectual generosity, based on their analysis of the case studies:

We have seen that this virtue is a glad willingness to give intellectual goods, both intrinsic and extrinsic, to others, and that this willingness is based on a dominance of two kinds of concerns: an interest in the intrinsic intellectual goods of knowledge, information, confirmation (or disconfirmation) of hypotheses, understanding, and other such goods; and an interest in the intellectual well-being of other people. In particular, these two kinds of concerns dominate over the concern to have, for oneself, such extrinsic intellectual goods as position, honors and wealth (Roberts and Wood, 2007, 304).

While Roberts and Wood pointed, in the above quote, to two concerns that ground the glad willingness to give intellectual goods, they also noted that there are examples of intellectual generosity in which only one concern is strong. One such exemplar is Rosalind Franklin (Roberts and Wood, 2007, 297-298). Franklin had made important progress towards determining the structure of DNA at the same time as Watson and Crick yet gave them credit happily and with no signs of disappointment when they were the ones to make the final discovery. ${ }^{3}$ Roberts and Wood suggested that, in Franklin's case, her willingness to give Watson and Crick credit happily and without resentment was based on a concern for intrinsic intellectual goods - any concern for Watson and Crick's well-being was "at most a side consideration" (Roberts and Wood, 2007, 298) because she did not care for them.

Finally, Roberts and Wood noted that intellectual generosity, like other virtues, is beneficial (Roberts and Wood, 2007, 301). For example, they suggested that McClintock's generosity in sharing her project with Creighton meant that: (i) the project progressed more quickly than if she had worked on it alone; (ii) McClintock had more time to work on additional projects and; (iii) Creighton was introduced to scientific life (Roberts and Wood, 2007, 302). Each of these, they suggested, led to the development of new intrinsic intellectual goods. Intellectual generosity is therefore important to scientific progress.

\section{William Thurston}

\subsection{Mathematicians on Thurston's Generosity}

William Thurston $(1946$ - 2012) worked primarily in geometry and topology. He was well known for thinking about mathematics in an original way and for making surprising con-

\footnotetext{
${ }^{3}$ Although Franklin didn't know this, her own data had been crucial to Watson and Crick's work, but they had used it without her knowledge and without giving her credit (Roberts and Wood, 2007, 298).
} 
This is a preprint of an article published in Synthese. The final authenticated version is available online at https://doi.org/10.1007/s11229-020-02660-w

nections between fields (Gabai and Kerckhoff, 2015). In many obituaries and memorials, his colleagues also praised his generosity. For example:

Bill was intellectually generous. He always had time to discuss mathematics with any interested person (Benham, 2011, 4).

Generally very generous with his time and ideas, Thurston had twenty-nine PhD students from his Princeton period ... (Gabai and Kerckhoff, 2015, 1321).

Over the years I often found Bill to be similarly generous with his time and his mathematical ideas.

$[\ldots]$

Bill was also generous with his deep knowledge of mathematics (Gabai et al., $2016,32)$.

Thurston was very generous with his ideas (Gabai, 2013).

I argue in section (3.4) that Thurston's generosity matches up with the kind of intellectual generosity analyzed by Roberts and Wood. To do this, I first describe two episodes from Thurston's career, one concerning the theory of foliations in section (3.2) and the other centering on the geometrization conjecture in section (3.3). Thurston's experiences while working in the theory of foliations serve as background context for his handling of the geometrization conjecture, which will be the focus of my discussion of his intellectual generosity. I do not discuss any of the mathematical details in what follows, but rather focus on Thurston's recollections of events as described in his "On proof and progress in mathematics." As Thurston's own recollections may be unintentionally biased, however, I supplement them with email interviews of his colleagues in section (3.4).

\section{$3.2 \quad$ Foliation Theory}

Thurston started working on foliation theory as a graduate student and proved a number of important theorems in the area. However, within a short period of time, mathematicians started leaving the field. While it might be natural to think that this was because Thurston had already proven all of the important theorems, he explicitly denied that this was the case: "I do not think that the evacuation occurred because the territory was intellectually exhausted - there were (and still are) many interesting questions that remain and that are probably approachable" (Thurston, 1994, 173). Instead, Thurston believed that two other factors were responsible for the mass withdrawal from the field: (i) the high cost of learning the theory; (ii) the need for researchers to obtain "credit" by proving new theorems.

Regarding the high cost of learning the theory, Thurston explained:

$[T]$ he results I proved (as well as some important results of other people) were documented in a conventional, formidable mathematician's style. They depended heavily on readers who shared certain background and certain insights. The theory of foliations was a young, opportunistic subfield, and the background was not standardized. I did not hesitate to draw on any of the mathematics I 
This is a preprint of an article published in Synthese. The final authenticated version is available online at https://doi.org/10.1007/s11229-020-02660-w

had learned from others. The papers I wrote did not (and could not) spend much time explaining the background culture. They documented top-level reasoning and conclusions that I often had achieved after much reflection and effort. I also threw out prize cryptic tidbits of insight, such as "the Godbillon-Vey invariant measures the helical wobble of a foliation", that remained mysterious to most mathematicians who read them. This created a high entry barrier: I think many graduate students and mathematicians were discouraged that it was hard to learn and understand the proofs of key theorems (Thurston, 1994, 173-174).

As Thurston emphasized, above, the high cost of learning the theory meant that it was difficult for mathematicians to obtain the intrinsic good of understanding. And, without understanding, it was nearly impossible for them to prove new theorems in the field. This meant that they struggled to obtain the extrinsic good of what Thurston called theoremcredits, i.e. the professional recognition that comes from proving new theorems:

When I started working on foliations, I had the conception that what people wanted was to know the answers. I thought that what they sought was a collection of powerful proven theorems that might be applied to answer further mathematical questions. But that's only part of the story. More than the knowledge, people want personal understanding. And in our credit-driven system, they also want and need theorem-credits (Thurston, 1994, 174, emphasis added).

This difficulty in extracting both intrinsic and extrinsic goods from the theory of foliations no doubt negatively affected the intellectual well-being of mathematicians working in the field. It is thus unsurprising that many left to pursue more intellectually enriching activities.

Equally unsurprising is the fact that the theory of foliations and other, related, fields of mathematical research suffered as a result of its abandonment. For example, Thurston described a "damper" being put on the theory (Thurston, 1994, 173). And while acknowledging that mathematicians continued to make progress in other, related areas, he also claimed that this progress "would have been much accelerated had mathematicians continued to pursue foliation theory vigorously" (Thurston, 1994, 173).

Although the situation that arose in foliation theory was unfortunate, Thurston reported that he learned from his experience and took steps to avoid a similar situation when working on the geometrization conjecture. And, as I will argue below, Thurston exemplified intellectual generosity in doing so.

\subsection{The Geometrization Conjecture}

Thurston (1982) made a famous conjecture about the geometric structure of mathematical entities called 3-manifolds, which became known as the geometrization conjecture. Further, he proved it for a special case - for a type of 3-manifold called a Haken manifold. ${ }^{4}$ However, Thurston had a very unusual way of thinking about the subject matter. As he explained

\footnotetext{
${ }^{4}$ Perelman $(2002,2003 \mathrm{a}, \mathrm{b})$ proved the full geometrization conjecture, which included the Poincaré conjecture.
} 
This is a preprint of an article published in Synthese. The final authenticated version is available online at https://doi.org/10.1007/s11229-020-02660-w

"some concepts that I use freely and naturally in my personal thinking are foreign to most mathematicians I talk to." (Thurston, 1994, 174). This, in turn, made it difficult for him to explain his approach to the subject matter in general, the meaning and importance of his conjecture, and the full details of his proof of the special case, to other mathematicians. As he put it "It was hard to communicate - the infrastructure was in my head, not in the mathematical community" (Thurston, 1994, 175).

Thurston was keen to avoid a repeat of what had happened in foliation theory, where the high cost of learning the theory combined with the need for theorem-credits led to the near abandonment of the field. Thus he focused his efforts on communicating the important "infrastructure" to the mathematical community. He explained "In reaction to my experience with foliations and in response to social pressures, I concentrated most of my attention on developing and presenting the infrastructure in what I wrote and in what I talked to people about" (Thurston, 1994, 176).

However, there was another, more secretive, component to Thurston's efforts: he was deliberately slow in publishing his theorems and proofs so that other researchers had time to publish their own and receive recognition for doing so. He explained "By concentrating on building the infrastructure and explaining and publishing definitions and ways of thinking but being slow in stating or in publishing proofs of all the "theorems" I knew how to prove, I left room for many other people to pick up credit" (Thurston, 1994, 176, emphasis added). In other words, Thurston claimed he was publishing his theorems and proofs deliberately slowly as a way to help other researchers extract extrinsic goods in the form of theoremcredits from the field.

Moreover, Thurston claimed that helping mathematicians to obtain both intrinsic and extrinsic goods led to new mathematical developments, i.e. to new intrinsic intellectual goods. He reported "There has been room for people to discover and publish other proofs of the geometrization theorem [for Haken manifolds]. These proofs helped develop mathematical concepts which are quite interesting in themselves, and lead to further mathematics" (Thurston, 1994, 176).

\subsection{Thurston's Intellectual Generosity}

We saw, in section (2), that intellectual generosity is understood by Roberts and Wood as "a glad willingness" to give intrinsic goods, such as understanding, and extrinsic goods, such as credit, to other researchers. Furthermore, this glad willingness is grounded in a concern for the intellectual well-being of other researchers and/or in a concern for intrinsic intellectual goods over and above a concern for one's own extrinsic intellectual goods.

Thurston's recollections from "On proof and progress in mathematics" certainly portray him as willing to give intrinsic and extrinsic goods to other mathematicians. Recall that he spoke of communicating important tools for thinking about 3-manifolds to other mathematicians and publishing his theorems and proofs deliberately slowly so that others had the opportunity to publish their own. However because reflections on our own experiences can be unintentionally biased, I contacted five prominent mathematicians who knew and worked with Thurston to ask whether this description is accurate: Ian Agol, Yakov Eliashberg, Joel Hass, Curtis McMullen and Yair Minsky.

The mathematicians I interviewed confirmed that the description is indeed fairly accu- 
This is a preprint of an article published in Synthese. The final authenticated version is available online at https://doi.org/10.1007/s11229-020-02660-w

rate. ${ }^{5}$ However, Minsky indicated that, at least initially, Thurston's slowness in publishing his theorems and proofs didn't help others to receive theorem-credits: "In practice it didn't at first quite work as he says he intended: there was so much material that "Thurston already knew" that it was hard to get credit for figuring it out" (Minsky, 2019). Nonetheless, he reported that the strategy of publishing slowly was eventually successful "Over time it did work. Lots of people entered the field and things were very interesting for quite a while" (Minsky, 2019).

Additionally Hass reported that Thurston was happy to give intrinsic and extrinsic intellectual goods in a slightly different way: by sharing ideas without asking for credit in return. He explained:

[Thurston] provided numerous mathematicians with key insights that propelled their individual research programs. He was extremely generous with this sharing of mathematical ideas. If he had been more aggressive in demanding credit for his ideas, he could have become a coauthor of dozens of important research papers. Instead many papers that depend to various extents on his insights either thank him for helpful conversations or don't mention his input at all" (Hass, 2019).

In summary, then, it is fair to say that Thurston was "gladly willing" to give intrinsic and extrinsic goods to his fellow mathematicians.

To show that Thurston was intellectually generous in Roberts and Wood's sense, however, we must also show that his willingness to give intellectual goods to other mathematicians was grounded in a concern for their intellectual well-being and/or in a concern for intrinsic intellectual goods over any concern for his own extrinsic intellectual goods. In fact, we will see that Thurston's willingness to give intellectual goods was grounded in both concerns.

Thurston's own recollections suggest that his willingness to give intellectual goods to other mathematicians was based on a concern for their intellectual well-being. As we saw, Thurston described how his experiences with foliation theory helped him realize that other mathematicians needed to gain understanding and an ability to obtain theorem-credits. In other words, he realized that they required both intrinsic and extrinsic goods for their intellectual well-being. Thurston then explicitly described how this led him to focus his efforts on communicating his ways of thinking, which gave his fellow mathematicians intrinsic intellectual goods, and to take his time publishing theorems and proofs, which gave others the opportunity to gain extrinsic intellectual goods. This meant Thurston received less extrinsic rewards himself, thus indicating his concern with the intellectual well-being of other mathematicians outweighed any concern he had for extrinsic intellectual goods.

I asked Agol, Eliashberg, Hass and Minsky whether it was fair to say that Thurston was concerned with the intellectual well-being of other mathematicians and they all confirmed that it was. ${ }^{6}$ However, some of the mathematicians I interviewed indicated that it is difficult

\footnotetext{
${ }^{5}$ There were, however, some doubts about the reasons Thurston was slow at publishing his theorems and proofs. More on this below.

${ }^{6}$ Nonetheless, some noted that Thurston was not perfect. For example, he would sometimes miss the deadlines for submitting letters or reports that were needed for job applications or promotions and did not take an active approach to advising students.
} 
This is a preprint of an article published in Synthese. The final authenticated version is available online at https://doi.org/10.1007/s11229-020-02660-w

to know for sure whether Thurston published his results slowly out of a concern for the intellectual well-being of others. For example, some suggested that there may be other contributing factors to his publication speed, such as a desire to focus on other activities. Nonetheless, it seems reasonable to suggest that his concern with the intellectual well-being of other mathematicians grounded his willingness to give them intrinsic intellectual goods and at least partially grounded his willingness to give them extrinsic goods.

Thurston's description of his experiences suggests that his willingness to give intellectual goods to others was also based on a concern for intrinsic intellectual goods. For example, in "On proof and progress in mathematics," he emphasized the importance of intrinsic intellectual goods, like understanding, over extrinsic ones:

I think that our strong communal emphasis on theorem-credits has a negative effect on mathematical progress (Thurston, 1994, 172).

What we are producing is human understanding. We have many different ways to understand and many different processes that contribute to our understanding. We will be more satisfied, more productive and happier if we recognize and focus on this (Thurston, 1994, 172-173).

Similarly, in the top rated answer to a MathOverflow question, Thurston emphasized the importance of understanding and learning over priority and credit:

In short, mathematics only exists in a living community of mathematicians that spreads understanding and breathes life into ideas both old and new. The real satisfaction from mathematics is in learning from others and sharing with others. All of us have clear understanding of a few things and murky concepts of many more. There is no way to run out of ideas in need of clarification. The question of who is the first person to ever set foot on some square meter of land is really secondary (Thurston, 2010).

I asked Agol, Eliashberg, Hass and Minsky if they could say more about Thurston's views on the importance of understanding vs. theorem-credits. Agol confirmed that Thurston cared deeply about understanding and gave a variety of examples from personal experience illustrating this. Hass reported that there was "[n]ot really much to add beyond what [Thurston] wrote" (Hass, 2019). Eliashberg emphasized the importance of understanding to mathematical progress: "[P]rogress in mathematics is measured not only in proven theorems, but also, for instance, in asking the right questions or creating new ways of seeing things, observing analogies" (Eliashberg, 2019). He added "I think that Bill rightly felt that beyond-theorems credits are much harder to get, especially for young mathematicians" (Eliashberg, 2019). Minsky agreed with Thurston about the importance of understanding over theorem-credits, but noted that theorem-credits have their uses: "I think [Thurston] is right about this, but that it is not an easy problem to solve. Theorem-credits are easier to keep track of than the broader things such as scholarly depth or general contribution to understanding" (Minsky, 2019). In sum, then, it seems fair to take Thurston's emphasis on the importance of understanding over theorem-credits at face value.

Thurston's concern with intrinsic intellectual goods plausibly grounded his willingness to give intellectual goods to other mathematicians. This is because he knew that "with no 
This is a preprint of an article published in Synthese. The final authenticated version is available online at https://doi.org/10.1007/s11229-020-02660-w

practitioners to keep it alive and to make it grow" (Thurston, 1994, 177) a theory would not generate intrinsic intellectual goods. And, as we saw earlier, Thurston realized that practitioners need to gain understanding and have the opportunity to publish their own work if they are to continue working in the field and keep it flourishing.

Thurston's own reflections as well as the testimony of mathematicians who knew and worked with him thus show that he was concerned both with intrinsic intellectual goods and with the intellectual well-being of other mathematicians. Furthermore, these concerns grounded his willingness to give intrinsic and extrinsic intellectual goods to others. Thurston was therefore intellectually generous in a way that matches Roberts and Wood's analysis.

Roberts and Wood also argued that intellectual generosity has the advantage of spurring the development of new intrinsic intellectual goods. We see this reflected in the case of Thurston as well. Recall Thurston reported that by giving intellectual goods to his fellow mathematicians he created an environment in which they were able to publish different proofs of the geometrization theorem for Haken manifolds and that their proofs led to new and interesting mathematical ideas. Agol, Hass, McMullen and Minsky confirmed that, for example, McMullen (1990) and Morgan and Shalen (1984, 1988a,b) published (parts of) proofs of Thurston's geometrization theorem for Haken manifolds. Furthermore, Agol reported that the work of Morgan and Shalen introduced the concepts of laminations and R-trees, which were generalized and became important in the field of 3-manifold topology. He also noted that McMullen's paper on the geometrization theorem was related to his proof of Kra's Theta Conjecture. McMullen reported in an interview with the Harvard Math Club that this was one of the projects for which he was awarded the fields medal (Oreskovich and Sagalovskiy, n.d.). As Agol summarized the situation: "the common theme here is that they [McMullen, Morgan and Shalen] each developed different tools to reprove Thurston's theorem which in turn had applications to other topics" (Agol, 2019).

Thurston's intellectual generosity, like McClintock's, thus generated high quality intrinsic intellectual goods.

\section{Reflections on Thurston \& Intellectual Generosity}

Having presented Roberts and Wood's account of intellectual generosity and argued that their analysis captures the sense in which Thurston was intellectually generous, I now want to consider intellectual generosity more deeply. To do this, I explore below four issues relating to Thurston's generosity. ${ }^{7}$

First, Thurston's (1994) paper was a response to an essay by Jaffe and Quinn (1993), who described Thurston's handling of the geometrization theorem for Haken manifolds in the following unflattering terms "A grand insight delivered with beautiful but insufficient hints, the proof was never fully published. For many investigators this unredeemed claim became a roadblock rather than an inspiration" (Jaffe and Quinn, 1993, 8). In other words, Thurston's deliberately slow pace of publishing meant that he never published a complete proof of the geometrization theorem for Haken manifolds. ${ }^{8}$ And this, claim Jaffe and Quinn, was harmful to other mathematicians. Given the interviews I conducted with mathematicians who knew

\footnotetext{
${ }^{7} \mathrm{I}$ am grateful to two anonymous reviewers for raising the issues considered below.

${ }^{8}$ Thurston did, however, publish parts of it (Thurston, 1994, 176).
} 
This is a preprint of an article published in Synthese. The final authenticated version is available online at https://doi.org/10.1007/s11229-020-02660-w

and worked with Thurston, Jaffe and Quinn's claim appears to be wrong, at least in the long run-recall that Minsky noted that Thurston's approach did eventually work and "[1]ots of people entered the field and things were very interesting for quite a while" (Minsky, 2019).

Nonetheless, Jaffe and Quinn's claim raises an interesting question: If publishing his theorems and proofs deliberately slowly had harmed, rather than helped, other mathematicians, could we still legitimately say that Thurston was intellectually generous? I suggest that we can. First, Thurston still happily gave intrinsic intellectual goods to others and he tried, though in this hypothetical case failed, to give them extrinsic goods. His (attempted) giving of these goods was still based on a concern for the intellectual well-being of others and on a concern for intrinsic intellectual goods. So he still fits well with the characterization of intellectual generosity given by Roberts and Wood.

Perhaps, however, we might think that Roberts and Wood's account is defective if it still judges Thurston as intellectually generous in this hypothetical case. Nonetheless I think that their account is correct in this regard. Indeed, we do not need to be perfect in order to be virtuous. For example, in discussing the moral virtue of generosity, Christian Miller wrote "Perfect generosity is an ideal that we likely cannot attain. But minimal or moderate possession of the virtue of generosity is attainable and is compatible with some mistakes and miscues" (Miller, 2018, 234, emphasis added). Miller also quoted Hunt as saying "The fact remains that a fully generous act may be utterly misguided and miscarried" (Miller, 2018, Hunt quoted on 235). So if Thurston believed that publishing his theorems and proofs deliberately slowly would benefit others by giving them extrinsic intellectual goods but had been mistaken in this belief, he can still legitimately be said to exemplify intellectual generosity.

Secondly, as Roberts and Wood noted, "[p]aradigmatically, generosity is a disposition to give property. But only the extrinsic intellectual goods have the property of being potential property" (Roberts and Wood, 2007, 293-294). They admit that this "requires the analysis of intellectual generosity to diverge in some respects from the paradigm case" (Roberts and Wood, 2007, 293). We might push this further and ask whether the giving of intrinsic intellectual goods could ever count as generous, since when we give such goods to other people we still get to keep them ourselves. However, sometimes giving intrinsic intellectual goods to others requires us to expend significant effort and give up considerable time to ensure they are successfully received.

For example, Thurston had to build up the community's knowledge of his tools for thinking about 3-manifolds from scratch. This took considerable time and effort. For example, he wrote:

I concentrated most of my attention on developing and presenting the infrastructure in what I wrote and in what I talked to people about (Thurston, 1994, 176, emphasis added)

By concentrating on building the infrastructure and explaining and publishing definitions and ways of thinking but being slow in stating or in publishing proofs of all the "theorems" I knew how to prove I left room for many other people to pick up credit (Thurston, 1994, 176, emphasis added)

More generally, Thurston noted earlier in his paper that while mathematicians can communicate effectively and efficiently with others who work in the same research area, 
This is a preprint of an article published in Synthese. The final authenticated version is available online at https://doi.org/10.1007/s11229-020-02660-w

communication between mathematicians who work in different fields is much harder and takes much more time (see Thurston (1994, sec. 3)). Thus successfully giving intrinsic intellectual goods from one field to researchers who work primarily in another will also require considerable time and effort.

Time and effort are much more like property than intrinsic intellectual goods. For example, once you have spent your time and effort performing a particular activity, it is gone and you cannot get it back. Thus if giving intrinsic intellectual goods requires you to donate a significant amount of your time and effort, then it seems that intellectual generosity is not so different from paradigmatic cases of moral generosity. For example, many paradigmatic examples of moral generosity considered in Miller (2018) involve the donation of a person's time.

Thirdly, we may note that in paradigmatic cases, generosity is motivated by a desire to help others. As intellectual generosity involves a concern with intrinsic over extrinsic intellectual goods, it may thus seem that some motivation in the intellectual case comes from a desire to see the field flourish. Perhaps, then, we might see mathematics itself as the beneficiary of some of Thurston's intellectual generosity. But mathematics may seem too abstract to be the recipient of genuine generosity, intellectual or otherwise. Thus we may wonder whether the virtue Thurston exemplifies can legitimately be called generosity.

In response to this, we can note that paradigmatic cases of generosity often involve giving to organizations or institutions. For instance, Miller (2018) considers a large donation to a university as an example of a generous act and describes the university as the "recipient" (Miller, 2018, 242) of such generosity. If an organization or institution can be the recipient of generosity, then why not a discipline like mathematics? It might be objected that in such cases the organization or institution isn't really the recipient of the generosity. For example, in the case of the university donation, perhaps it is the students, faculty and staff who are the recipients of the generosity, rather than the university itself. But something similar can be said for mathematics. In particular, mathematicians will benefit intellectually from the flourishing of mathematics and so perhaps it is mathematicians who are the real recipients of Thurston's intellectual generosity rather than mathematics itself.

Finally, we might think that, while not concerned with receiving credit, Thurston could have worked to help other mathematicians understand his ways of thinking because he did not want to be alone in his field. And if he was primarily concerned with avoiding intellectual loneliness and isolation, perhaps we should not be willing to describe him as intellectually generous.

In response to this, I think it is safe to say that Thurston did not want to be alone in his field. Indeed he admits "most mathematicians don't like to be lonely, and they have trouble staying excited about a subject, even if they are personally making progress, unless they have colleagues who share their excitement" (Thurston, 1994, 171). However, Thurston was not primarily concerned with avoiding loneliness and isolation. Instead, his desire to not be alone in his field was closely tied up with his recognition that a field needs practitioners if it is to continue to thrive. Indeed, he remarked that one of the two worst possible outcomes of the episode with the geometrization conjecture would have been "[f]or me to present an unassailable and hard-to-learn theory with no practitioners to keep it alive and to make it grow" (Thurston, 1994, 177, emphasis added). Recall that he also remarked "mathematics only exists in a living community of mathematicians that spreads 
This is a preprint of an article published in Synthese. The final authenticated version is available online at https://doi.org/10.1007/s11229-020-02660-w

understanding and breathes life into ideas both old and new" (Thurston, 2010).

Thurston's desire to not be alone in a discipline thus comes from a concern with the health of that field and the recognition that, if he were alone in it, far fewer intrinsic intellectual goods would be generated. In other words, it wasn't a fear of loneliness or isolation, but rather his concern for intrinsic intellectual goods, that grounded his giving of intellectual goods to other mathematicians. And giving to others out of a concern for the continued development of the field does indeed seem intuitively generous.

\section{The Benefits of Intellectual Generosity: Overcoming Prob- lems with the Reward Structure of Mathematics}

Although Roberts and Wood indicated that intellectual generosity promotes the development of new intrinsic intellectual goods, they didn't say much about how it promotes this. ${ }^{9}$ Here I suggest that intellectual generosity can promote the production of intrinsic intellectual goods by offsetting problems caused by the reward structure of mathematics. ${ }^{10}$ In this section, I thus first examine the reward structure of mathematics and argue that it can sometimes stymie mathematical progress. I then show how intellectual generosity can help ameliorate these problems and thus generate intrinsic intellectual goods.

As we have seen in the discussion of the Thurston case study, the reward structure of mathematics is based on theorem-credits. That is, mathematicians receive extrinsic goods in the form of recognition and rewards for proving new theorems. Further, the reward structure of mathematics is similar to that of science in that there is a priority rule: if two mathematicians, $M$ and $N$, are working on proving the same theorem and $M$ proves it first, it is $M$ who gets the credit, even if $N$ proves it only a few days after $M$. The history of mathematics illustrates the existence of such a priority rule with examples of controversies over which mathematician made a particular discovery first and should therefore receive credit. Examples include the famous dispute between Newton and Leibniz over calculus (Hellman, 2006, chp.3), Bolyai's claim that Gauss plagiarized his discovery of non-Euclidean geometry (Waldo Dunnington et al., 2004, 188), and an argument between father and son Johann and Daniel Bernoulli over hydrodyanmics, with Johann trying to take credit for his son's work by predating his book (Hellman, 2006, chp.4)!

Economists of science have argued that the priority rule in science "encourages the production and sharing of knowledge" (Stephan, 2012, chp.1), or, in Roberts and Wood's terminology, intrinsic intellectual goods. More precisely, they argue that since knowledge and other intrinsic goods are very much like public goods, scientists face a free rider problem (Stephan, 2012, chp.1). In the absence of the priority rule, production of knowledge and other intrinsic goods slows or stops. Further, philosophers of science have argued that the reward structure is beneficial by ensuring an optimal division of cognitive labor (Strevens,

\footnotetext{
${ }^{9}$ Roberts and Wood did note that a concern with intrinsic over extrinsic intellectual goods characteristic of intellectual generosity can help researchers persevere where others might give up and so potentially generate new intrinsic intellectual goods in this manner (Roberts and Wood, 2007, 301-302). They also pointed to specific ways in which McClintock's intellectual generosity to Creighton generated intrinsic intellectual goods (Roberts and Wood, 2007, 302).

${ }^{10} \mathrm{I}$ am grateful to an anonymous reviewer for pointing out relevant connections between intellectual generosity and social epistemology.
} 
This is a preprint of an article published in Synthese. The final authenticated version is available online at https://doi.org/10.1007/s11229-020-02660-w

2003) and by incentivizing researchers to share their intermediate results (Heesen, 2019).

However the reward structure of science is not without its problems (see e.g. Heesen (2018), Zollman (2019)). Similarly, the reward structure of mathematics has drawbacks. In particular, there are two ways in which it serves to stymie the production of intrinsic intellectual goods. First, superstars in a field, like Thurston, will obtain the vast majority of theorem-credits thanks to the priority rule. In some cases, as we saw with foliation theory, this can result in the abandonment of the field and thus the cessation of production of intrinsic goods. Second, mathematicians receive credit for proving theorems, not for other important activities like making results, concepts and methods more accessible to mathematicians working in other areas. However such activities are important for the development of high quality intrinsic intellectual goods. The disincentive to partake in them can thus significantly stymie the production of intrinsic goods. Below I will consider each of these problems in more detail and argue that cultivating intellectual generosity is one way in which they can be overcome.

\subsection{Superstars Taking All the Credit}

Mathematical superstars, like Thurston, are likely to discover and publish proofs of new theorems before other mathematicians. When there is a small number of superstars working in a given field, the superstars will then collectively receive most of the theorem-credits in the area, thanks to the priority rule. However, other mathematicians still need to obtain their own theorem-credits in order to get a job or to be promoted. As they have little chance of competing against the superstars in the field, non-superstars will likely look to join a different field where they have more chance of obtaining theorem-credits. As the number of non-superstars working in the field diminishes, the superstars will likely move to other fields as well. Indeed, Thurston reported that "fairly soon [after mathematicians started leaving foliation theory], I turned to other interests as well" (Thurston, 1994, 173). The superstars may change fields because it is not fun, for example, to compete for theoremcredits when there are few others to compete against, or to make exciting discoveries when there are few others to share them with. The field thus becomes abandoned and further production of intrinsic intellectual goods in the area stops. While it has been argued that the reward structure of science can help ensure a favorable division of cognitive labor (see e.g. Strevens (2003), Thoma (2015)), we thus see here that the theorem-credit economy can lead to a failure to divide cognitive labor between fields.

This unfortunate situation can be avoided, however, if superstars cultivate intellectual generosity. Suppose that, like Thurston, the superstars decide to publish their theorems and proofs deliberately slowly because they are concerned with the intellectual well-being of their fellow researchers and with the intrinsic goods of their field. Other mathematicians will then have the opportunity to find and publish their own proofs first and thus obtain the theorem-credits that they need. The non-superstars will then no longer be pressured to leave the field and so the production of intrinsic intellectual goods can continue.

\subsection{The Disincentive to Make Work Accessible}

As we have seen, mathematicians are awarded credit for proving new theorems. They are not, however, awarded credit for other activities that make work from one field more 
This is a preprint of an article published in Synthese. The final authenticated version is available online at https://doi.org/10.1007/s11229-020-02660-w

accessible to researchers in other fields, such as exposition. ${ }^{11}$ Exposition can include, for example, writing review articles, a book providing an overview of the field or even blog posts. As a concrete example, Thurston engaged in exposition when he focused on communicating important tools from 3-manifolds to the wider mathematical community. In particular, he wrote a set of lecture notes that were widely circulated whose "intent is to describe the very strong connection between geometry and lowdimensional topology in a way which will be useful and accessible (with some effort) to graduate students and mathematicians working in related fields, particularly 3-manifolds and Kleinian groups" (Thurston, 2002, iii, emphasis added).

The lack of reward for expository work in mathematics has been noted by a number of researchers. For example, Krantz commented "American mathematics does not prize expository work as highly as do other American sciences, nor as highly as does European mathematics" (Krantz, 1997, 197). And while Rota titled a section of his (1997) paper "You are more likely to be remembered by your expository work" (Rota, 1997b, 199), being remembered is not necessarily the same as being rewarded. Indeed, elsewhere Rota remarked "high-caliber expository work is more exploited than rewarded by the mathematical community" (Rota, 1997a, 175). Finally the Society for Industrial and Applied Mathematics noted that while one of their journals publishes review articles "such articles seem to be more highly regarded (and hence rewarded) in other disciplines" (Crowley, 2014).

Nonetheless, although it is not rewarded, exposition is difficult work. Recall that Thurston noted the difficulty of communicating ideas to mathematicians working in different areas (Thurston, 1994, sec.3). Further, as Krantz put it "You really need to be a master of your subject and have a very broad perspective and genuine insight to write a good expository piece" (Krantz, 1997, 197).

As making work in one field more accessible to mathematicians working in other fields is not rewarded and is difficult, mathematicians are thus strongly disincentivized to do it. Yet making work accessible to other mathematicians is important for the generation of high quality intrinsic goods. Indeed, making a field more accessible attracts new researchers. When this happens, more researchers are available to spend their time and effort tackling research problems in the field, which plausibly means that more such problems will be solved, and thus intrinsic intellectual goods generated, in a shorter time frame than before.

However there is another way in which making a field more accessible can help generate new intrinsic intellectual goods: by helping mathematicians to make connections between fields. This is especially important given that (i) making connections between different fields is becoming increasingly important to mathematical progress; and (ii) mathematics has become so specialized that it is hard for mathematicians to make such connections. I will substantiate both points (i) and (ii) below before arguing how intellectual generosity can help.

\subsubsection{The importance of finding connections between mathematical fields}

Mathematicians have drawn attention to the fact that making connections between different fields can facilitate, and is sometimes required for, the solution of mathematical problems

\footnotetext{
${ }^{11}$ The arguments that I make in this section are a modified and shortened form of arguments I make in Morris (n.d.).
} 
This is a preprint of an article published in Synthese. The final authenticated version is available online at https://doi.org/10.1007/s11229-020-02660-w

and thus for the attainment of new intrinsic intellectual goods. For example:

Making unexpected connections between apparently unrelated areas of mathematics often lies at the heart of solving a great problem (Stewart, 2013, 44).

[M]any of the results I proved [in abelian group theory] that most impressed other mathematicians were obtained very cheaply, by using results that I knew from fields that seemed far removed from abelian group theory and which most abelian group theorists either weren't aware of, or at least had never paid much attention to (Lady, n.d.).

One characteristic that most of the failed attempts [to prove the Poincaré conjecture, a now proven theorem in topology] share is a reliance on topological arguments. But, noted John Morgan of Columbia University, "It seems like this problem does not succumb to that type of argument." Rather, he said, one needs tools from outside topology (Jackson, 2006, 898).

The mystery as well as the glory of mathematics lie not so much in the fact that abstract theories do turn out to be useful in solving problems, but in that wonder of wonders, in the fact that a theory meant for one type of problem is often the only way of solving problems of entirely different kinds, problems for which the theory was not intended. These coincidences occur so frequently that they must belong to the essence of mathematics (Rota, 1991, 488).

A study conducted by the Committee on the Mathematical Sciences in 2025 further emphasizes the importance of finding connections between mathematical fields. For example, the authors of the study explained:

Based on testimony received at its meetings, conference calls with leading researchers $[\ldots]$, and the experiences of its members, the committee concludes that the importance of connections among areas of research has been growing over the past two decades or more. This trend has been accelerating over the past 10-15 years, and all indications are that connections will continue to be very important in the coming years (National Research Council, 2013, 93).

The authors of the study reported that connections between fields are important because: (i) to make progress, research now needs to draw upon multiple fields (National Research Council, 2013, 93); (ii) connections stimulate further research by pointing at "deeper relationships" (National Research Council, 2013, 96).

Making connections between mathematical fields is thus crucial to mathematical progress and the attainment of high quality intrinsic intellectual goods.

\subsubsection{How hyperspecialization makes finding connections harder}

Mathematics has become a highly specialized discipline. For example, the mathematician Piergiorgio Odifreddi writes: 
This is a preprint of an article published in Synthese. The final authenticated version is available online at https://doi.org/10.1007/s11229-020-02660-w

The problems that are simple and easy to solve are few, and once they have been solved a discipline can only grow by tackling complex and difficult problems, requiring the development of specific techniques, and hence of specialization. This is indeed what happened in the twentieth century, which has witnessed a hyperspecialization of mathematics that resulted in a division of the field into subfields of ever narrower and strictly delimited borders (Odifreddi, 2006, 2)

Further indication of the extent to which mathematics is hyperspecialized can be seen by consulting the 2010 Mathematics Subject Classification which provides codes used to classify mathematical work into different subdisciplines. For example 03 is the 2-digit code for the area Mathematical Logic and Foundations and 03A05 is the 5-digit code for the subdiscipline of Philosophical and critical. ${ }^{12}$ There are a total of 632 -digit codes which get further broken down into over 5,000 3-or-5-digit codes representing subdisciplines. (Dunne and Hulek, n.d.).

Such specialization has its benefits. For example, Casadevall and Fang list the benefits of specialization in science as "efficiency, reduced time to production, improved quality, and the partitioning of vast quantities of knowledge into more-manageable units" (Casadevall and Fang, 2014, 1356). Presumably the same or similar benefits apply to mathematics. Nonetheless the hyperspecialization of mathematics also has a significant drawback: it makes it difficult for mathematicians to become familiar with fields outside their primary area of expertise. For example, Thurston remarked:

Basic concepts used every day within one subfield are often foreign to another subfield. Mathematicians give up on trying to understand the basic concepts even from neighboring subfields, unless they were clued in as graduate students (Thurston, 1994, 166)

The mathematician Felix Browder made similar comments:

Browder also warned that mathematics was becoming too specialized and siloed, such that experts in different subfields, not to mention in different sciences, no longer shared a common language or mutual understanding (Lin, 2016)

Making it harder for mathematicians to become familiar with fields outside their primary research area makes it harder for them to make connections to other areas by themselves. Indeed, to find such connections between her primary research field and other areas, a mathematician needs to be familiar with those others areas!

However, it also makes it harder for mathematicians in one field to find connections by collaborating with mathematicians from other fields. For example, suppose you are working on a problem in one field and begin to realize that you need to make a connection to a different field to continue to make progress. ${ }^{13}$ You thus want to look for collaborators in other fields who can help you. However, you first need to figure out where to look for

\footnotetext{
${ }^{12}$ See https://mathscinet.ams.org/mathscinet/msc/msc2010.html for the full list.

${ }^{13}$ Such scenarios happen in practice: "Fields in the mathematical sciences are mature enough so that researchers know the capabilities and limitations of the tools provided by their field, and they are seeking tools from other areas" (National Research Council, 2013, 97).
} 
This is a preprint of an article published in Synthese. The final authenticated version is available online at https://doi.org/10.1007/s11229-020-02660-w

collaborators! In other words, you need to identify what other fields might provide the tools to help solve your problem. Presumably to do this, however, you need to have some knowledge and understanding of other mathematical fields, including, for example, their subject matter, general approach, and perhaps even some of their important concepts and methods. Further, once you have found a collaborator, you then need to be able to communicate and work together to successfully find a solution to your problem. This presumably requires that you each come to have some understanding of the concepts, methods and approaches from each field that are relevant to your problem.

In summary, then, hyperspecialization makes it difficult for mathematicians to find connections between fields, either working on their own or collaboratively, because it makes it harder for them to become familiar with fields other than their own primary research area.

\subsubsection{The Disincentive to Make Work Accessible Hinders the Attainment of Intrinsic Goods}

We have seen, above, that (i) making connections between fields is important to mathematical progress and the production of intrinsic intellectual goods; and (ii) hyperspecialization makes it harder for mathematicians to make these connections by making it more difficult for them to become familiar with fields other than their own. We have also seen that the reward structure of mathematics disincentivizes mathematicians from making their work accessible, e.g. via exposition. The reward structure therefore exacerbates the problem of hyperspecialization. Indeed, if mathematicians made their work more accessible by engaging in exposition, it would help mathematicians working in other research areas to become familiar with it and thus to potentially make connections between fields, either on their own or in collaboration with other researchers.

However the situation can be ameliorated if mathematicians cultivate intellectual generosity. More concretely, suppose that there is an intellectually generous mathematician whose primary research area is field $F$. She cares deeply about the intellectual well-being of other mathematicians and has a great concern for intrinsic intellectual goods. She thinks that mathematicians working in different fields would benefit intellectually from knowing the subject matter, general approach and important concepts, methods and results of $F$. She also thinks mathematics as a whole would benefit if more mathematicians were familiar with at least the basics of $F$, since it may lead to connections between and breakthroughs in $F$ and other fields. Like Thurston, she thus decides to communicate the subject matter, general approach and important tools of $F$ to mathematicians in other fields by engaging in high quality expository work. If she succeeds, mathematicians may make more breakthroughs in her own field $F$ as well as other mathematical areas by making connections between them. This would then create high quality intrinsic intellectual goods.

\subsection{Summary}

Although economists and philosophers of science have argued that the reward structure of science has important benefits, we have seen that the theorem-credit economy in mathematics can sometimes stymie the production of intrinsic intellectual goods. One of the benefits 
This is a preprint of an article published in Synthese. The final authenticated version is available online at https://doi.org/10.1007/s11229-020-02660-w

of intellectual generosity is that it can overcome the difficulties caused by the reward structure and thus promote the production of intrinisic intellectual goods in mathematics.

It might be thought, however, that as the reward structure of mathematics can be changed, intellectual generosity is not that important to the production of intrinsic intellectual goods. For example, perhaps a change to the reward structure could ameliorate the problems described above, reducing the need for mathematicians to be intellectually generous. Nonetheless, determining how best to change the reward structure is a difficult and complex task. Indeed, in the case of science, the best reward structure with respect to one issue may conflict with the best for another, requiring trade-offs to be made (Heesen, 2019). So even if the reward structure of mathematics is changed, mathematicians will likely still need to cultivate intellectual generosity to ensure the continued development of intrinsic intellectual goods.

\section{Concluding Remarks}

In this paper I argued that the mathematician William Thurston exemplified intellectual generosity in Roberts and Wood's sense. More precisely, based on a concern for the intellectual well-being of others and a concern for intrinsic intellectual goods, Thurston happily gave both intrinsic and extrinsic intellectual goods to his fellow mathematicians. I further argued that intellectual generosity is beneficial because it can overcome problems caused by the theorem-credit economy in mathematics, thus promoting the production of intrinsic intellectual goods.

Mathematics has therefore proven to be a useful domain to consider when analyzing virtues like intellectual generosity. Interestingly, there are plenty of other prominent mathematicians who are praised for their intellectual generosity including Richard Dedekind, Paul Erdős, ${ }^{14}$ Leonhard Euler and Emmy Noether:

Another passage is worth quoting as an illustration ... of Dedekind's generous attitude toward alternative constructions ... (Stein, 1988, 257, note 26).

Erdős was not only an outstanding mathematician but a very kind and generous human being, who encouraged hundreds of mathematicians over the decades, especially young aspirants to the subject (Alladi, 1998, 5).

Although by disposition somewhat irascible, Euler was not quarrelsome; he was exceptionally generous, never once making a claim of priority and in some cases actually giving away discoveries that were his own (Truesdell, 2007, 30).

[I]t is in this area [foundations of algebraic geometry] that one of the typical incidents occurred which show E. Noether's generous attitude concerning the authorship of publications (Dick, 1981, 59).

It may prove fruitful to analyze and compare the intellectual generosity of these mathematicians to the intellectual generosity of Thurston.

\footnotetext{
${ }^{14}$ Thanks to Bonnie Gold for alerting me to Erdős's generosity.
} 
This is a preprint of an article published in Synthese. The final authenticated version is available online at https://doi.org/10.1007/s11229-020-02660-w

Acknowledgments I am very grateful to Ian Agol, Yakov Eliashberg, Yacin Hamami, Bonnie Gold, Joel Hass, Erich Kummerfeld, Curtis McMullen, Yair Minsky and Alan Weinstein. I am also very grateful to participants at both the Mathematics in Practice conference held at Stanford University in May 2019 and the MidWest Philosophy of Mathematics Workshop held at Notre Dame in November 2019. Finally I am very grateful to three anonymous reviewers for their many helpful comments and suggestions. This work was partly undertaken while I held a Postdoctoral Scholarship at the Suppes Center for History and Philosophy of Science at Stanford University.

\section{References}

Ian Agol. Personal communication via email, July 2019.

Krishnaswami Alladi. Editorial. The Ramanujan Journal, 2(1):5-6, March 1998.

Jason Baehr. Four varieties of character-based virtue epistemology. The Southern journal of philosophy, 46(4):469-502, December 2008.

Jason Baehr. The Inquiring Mind: On Intellectual Virtues and Virtue Epistemology. OUP Oxford, June 2011.

Heather Battaly. Virtue epistemology. Philosophy Compass, 3(4):639-663, July 2008.

Craig Benham. UC Davis mathematics newsletter, 2011. https://www.math.ucdavis. edu/files/6013/7176/9863/newsletter_2012-rev1-3.pdf Archived at https:// perma.cc/6LKD-S4UX.

Arturo Casadevall and Ferric C Fang. Specialized science. Infection and immunity, 82(4): 1355-1360, April 2014.

Ian M Church. The doxastic account of intellectual humility. Logos 8 Episteme, 7(4): 413-433, 2016.

Harriet B Creighton and Barbara McClintock. A correlation of cytological and genetical crossing-over in Zea mays. Proceedings of the National Academy of Sciences of the United States of America, 17(8):492-497, August 1931.

Jim Crowley. Putting a value on expository writing. https://sinews.siam. org/Details-Page/putting-a-value-on-expository-writing. Archived at https: //perma.cc/H4GL-9F74, 2014. Accessed: 2019-9-9.

Auguste Dick. Emmy Noether 1882-1935. Birkhäuser Boston, 1981.

Edward Dunne and Klaus Hulek. MSC2020. https://msc2020.org/ Archived at https: //perma.cc/BG4X-DPGW, n.d. Accessed: 2019-12-3.

Yakov Eliashberg. Personal communication via email, June 2019.

Miranda Fricker. Epistemic Injustice: Power and the Ethics of Knowing. Oxford University Press, 2007. 
This is a preprint of an article published in Synthese. The final authenticated version is available online at https://doi.org/10.1007/s11229-020-02660-w

David Gabai. Fine letters, 2013. https://www.math.princeton.edu/sites/default/ files/2017-10/newsletter2013_2.pdf Archived at https://perma.cc/J9XB-WYKF.

David Gabai and Steve Kerckhoff. William P. Thurston, 1946-2012. Notices of the American Mathematical Society., 62(11):1318-1332, December 2015.

David Gabai, Steven Kerckhoff, Yair Minsky, Lee Mosher, Jeff Weeks, Benson Farb, Danny Calegari, Ian Agol, Genevieve Walsh, Carol Wood, Tan Lei, and Curtis T McMullen. William P. Thurston, 1946-2012, part II. Notices of the American Mathematical Society., 63(01):31-41, January 2016.

John Greco. Virtues in epistemology. In The Oxford Handbook of Epistemology. Oxford University Press, New York, 2002.

Joel Hass. Personal communication via email, June 2019.

Remco Heesen. Why the reward structure of science makes reproducibility problems inevitable. The journal of philosophy, 115(12):661-674, December 2018.

Remco Heesen. The credit incentive to be a maverick. Studies in history and philosophy of science, 76:5-12, August 2019.

Hal Hellman. Great Feuds in Mathematics: Ten of the Liveliest Disputes Ever. Wiley, September 2006.

Allyn Jackson. Conjectures no more? Consensus forming on the proofs of the Poincaré and geometrization conjectures. Notices of the American Mathematical Society, 53(8): 897-901, September 2006.

Arthur Jaffe and Frank Quinn. Theoretical mathematics: Toward a cultural synthesis of mathematics and theoretical physics. Bulletin of the American Mathematical Society, 29 (1):1-14, November 1993.

Steven George Krantz. A Primer of Mathematical Writing: Being a Disquisition on Having Your Ideas Recorded, Typeset, Published, Read and Appreciated. American Mathematical Soc., 1997.

Lee Lady. How does one do mathematical research? (or maybe how not to), n.d. http:// www.math.hawaii.edu/lee/how-to.html. Archived at https://perma.cc/LZE6-ETTD.

Thomas Lin. Remembering Felix Browder, a nonlinear genius in a nonlinear world. The New Yorker, December 2016. Archived at https://perma.cc/UGA5-VQ38.

Curtis McMullen. Iteration on Teichmüller space. Inventiones Mathematicae, 99(1):425454, December 1990.

José Medina. The relevance of credibility excess in a proportional view of epistemic injustice: Differential epistemic authority and the social imaginary. Social Epistemology, 25(1):1535, January 2011. 
This is a preprint of an article published in Synthese. The final authenticated version is available online at https://doi.org/10.1007/s11229-020-02660-w

Christian B Miller. Generosity: A preliminary account of a surprisingly neglected virtue. Metaphilosophy, 49(3):216-245, April 2018.

Yair Minsky. Personal communication via email, July 2019.

John W Morgan and Peter B Shalen. Valuations, trees, and degenerations of hyperbolic structures, I. Annals of mathematics, 120(3):401-476, 1984.

John W Morgan and Peter B Shalen. Degenerations of hyperbolic structures, II: Measured laminations in 3-manifolds. Annals of mathematics, 127(2):403-456, 1988a.

John W Morgan and Peter B Shalen. Degenerations of hyperbolic structures, III: Actions of 3-manifold groups on trees and thurston's compactness theorem. Annals of mathematics, 127(3):457-519, 1988b.

Rebecca L. Morris. Increasing specialization: Why we need to make mathematics more accessible. Unpublished manuscript, n.d.

National Research Council. The Mathematical Sciences in 2025. The National Academies Press, 2013.

Piergiorgio Odifreddi. The Mathematical Century: The 30 Greatest Problems of the Last 100 Years. Princeton University Press, October 2006.

Anne-Marie Oreskovich and Dmitry Sagalovskiy. Math Club interview with Professor Curtis McMullen, n.d. http://www. math.harvard.edu/ ctm/expositions/html/interview. html. Archived at https://perma.cc/C9S7-N9SY.

Grisha Perelman. The entropy formula for the Ricci flow and its geometric applications. arXiv:math/0211159 [math.DG], November 2002. URL https://arxiv.org/abs/math/ 0211159.

Grisha Perelman. Ricci flow with surgery on three-manifolds. arXiv:math/0303109 [math.DG], March 2003a. URL https://arxiv.org/abs/math/0303109.

Grisha Perelman. Finite extinction time for the solutions to the Ricci flow on certain threemanifolds. arXiv:math/0307245 [math.DG], July 2003b. URL https://arxiv.org/abs/ math/0307245.

Robert C Roberts and W Jay Wood. Intellectual Virtues: An Essay in Regulative Epistemology. Oxford University Press, Oxford, 2007.

Gian-Carlo Rota. The concept of mathematical truth. The Review of metaphysics, 44(3): 483-494, 1991.

Gian-Carlo Rota. The phenomenology of mathematical beauty. Synthese, 111(2):171-182, May 1997a.

Gian-Carlo Rota. Ten lessons I wish I had been taught. In Gian-Carlo Rota and Fabrizio Palombi, editors, Indiscrete Thoughts, pages 195-203. Birkhäuser Boston, Boston, MA, 1997b. 
This is a preprint of an article published in Synthese. The final authenticated version is available online at https://doi.org/10.1007/s11229-020-02660-w

Ernest Sosa. Reliabilism and intellectual virtue. In Knowledge in Perspective: Selected Essays in Epistemology, pages 131-146. Cambridge University Press, March 1991a.

Ernest Sosa. Intellectual virtue in perspective. In Knowledge in Perspective: Selected Essays in Epistemology, pages 270-294. Cambridge University Press, March 1991b.

Howard Stein. Logos, logic, and logistiké: Some philosophical remarks on the nineteenth century transformation of mathematics. History and philosophy of modern mathematics, 11:238-259, 1988.

Paula Stephan. How Economics Shapes Science. Harvard University Press, 2012.

Ian Stewart. Visions of Infinity: The Great Mathematical Problems. Basic Books, 2013.

M Strevens. The role of the priority rule in science. The journal of philosophy, 100(2): $55-79,2003$.

Johanna Thoma. The epistemic division of labor revisited. Philosophy of science, 82(3): 454-472, 2015.

William Thurston. Geometry and topology of three-manifolds. Available online at http://library.msri.org/books/gt3m/, 2002.

William P Thurston. Three-dimensional manifolds, Kleinian groups and hyperbolic geometry. Bulletin of the American Mathematical Society, 6(3), 1982.

William P Thurston. On proof and progress in mathematics. Bulletin of the American Mathematical Society, 30:161-177, 1994.

William P Thurston. What's a mathematician to do? https://mathoverflow.net/ questions/43690/whats-a-mathematician-to-do/44213, 2010. Archived at https: //perma.cc/XVA8-8L6D.

Clifford Truesdell. Leonard Euler, supreme geometer. In William Dunhem, editor, The Genius of Euler: Reflections on His Life and Work, pages 13-42. MAA, 2007.

John Turri, Mark Alfano, and John Greco. Virtue epistemology. In Edward N Zalta, editor, The Stanford Encyclopedia of Philosophy. Metaphysics Research Lab, Stanford University, summer 2018 edition, 2018. https://plato.stanford.edu/archives/sum2018/ entries/epistemology-virtue/.

G Waldo Dunnington, Jeremy Gray, and Fritz-Egbert Dohse. Carl Friedrich Gauss: Titan of Science. MAA, October 2004.

Dennis Whitcomb, Heather Battaly, Jason Baehr, and Daniel Howard-Snyder. Intellectual humility: Owning our limitations. Philosophy and phenomenological research, 94(3):509539, May 2017.

Linda T Zagzebski. Virtues of the Mind. Cambridge University Press, September 1996.

Kevin J. S. Zollman. The scientific Ponzi scheme. Unpublished manuscript available on the PhilSci-Archive http://philsci-archive.pitt.edu/16264/, 2019. 